\title{
Drogas y rendimiento académico en estudiantes del área de salud de dos universidades latinoamericanas
}

\author{
Xóchitl De San Jorge-Cárdenas ${ }^{\star}$, Paulina Beverido-Sustaeta ${ }^{\star *}$, Betzaida Salas-García ${ }^{* * *}$, Alma \\ Cruz-Juárez ${ }^{* * * *}$, Marcela América Roa-Cubaque ${ }^{\star * * * *}$, Guiomar Haydee Rubiano-Díaz ${ }^{\star * * * *}$
}

\footnotetext{
* Doctora en Antropología.

Investigadora, Universidad

Veracruzana, miembro del cuerpo

académico Drogas y Adicciones:

Un Enfoque Multidisciplinario,

Xalapa, México. Correo electrónico:

xdesanjorge@uv.mx

* Magíster en Prevención del Consumo de Drogas. Investigadora, Universidad Veracruzana, miembro del cuerpo académico Drogas y Adicciones:

Un Enfoque Multidisciplinario,

Xalapa, México. Correo electrónico:

pbeverido@uv.mx

*** Magíster en Demografía. Investigadora Universidad Veracruzana, miembro del cuerpo académico Drogas y Adicciones: Un Enfoque Multidisciplinario, Xalapa, México. Correo electrónico: besalas@ uv.mx

**** Doctora en Psicología. Investigadora, Universidad Veracruzana, miembro del cuerpo académico Drogas y Adicciones: Un Enfoque Multidisciplinario, Xalapa, México. Correo electrónico: acruz@ uv.mx

$* * * * *$ Magíster en Tabaquismo. Investigadora Universidad de Boyacá, miembro del grupo de investigación Oxigenar, programa Terapia Respiratoria, Tunja, Colombia. Correo electrónico: maroa@uniboyaca.edu.co

* Magíster en Tabaquismo. Investigadora, Universidad de Boyacá, miembro del grupo de investigación Oxigenar, programa Terapia Respiratoria, Tunja, Colombia. Correo electrónico: guiru@ uniboyaca.edu.co
}

Recibido: 20 de junio del 2016

Aprobado: 7 de septiembre del 2017

Cómo citar este artículo: De San JorgeCárdenas, X., Beverido-Sustaeta, P., SalasGarcía, B., Cruz-Juárez, A., Roa-Cubaque, M. A. y Rubiano-Díaz, G. H. (2017). Drogas y rendimiento académico en estudiantes del área de salud de dos universidades latinoamericanas. Pensando Psicología, 13(22), 51-60. doi: https://doi.org/10.16925/ pe.v13i22.1988

\section{Resumen}

Introducción: el consumo de drogas en la población juvenil de los países en desarrollo se ha incrementado durante las últimas décadas; las instituciones educativas y de salud están preocupadas por esta tendencia, ya que puede alterar el aprendizaje y la memoria, por lo que afecta particularmente a los estudiantes universitarios. Objetivo: describir y comparar la relación entre consumo de drogas y rendimiento académico en estudiantes del área de Ciencias de la Salud de dos universidades latinoamericanas. Metodología: se utilizó un muestreo no probabilístico por conveniencia y se aplicó en línea el cuestionario Consumo de Drogas en Estudiantes Universitarios (CODEu). El análisis de la información se realizó con base en estadísticas descriptivas y se aplicó el coeficiente de correlación biserial puntual y la prueba no paramétrica de Kruskal-Wallis, tomando en cuenta el promedio de calificaciones obtenido durante el último periodo cursado. Resultados: en la Universidad Veracruzana se encontró que el consumo de alcohol, marihuana, cocaína e inhalables disminuye el rendimiento académico, mientras que en la Universidad de Boyacá solo el consumo de tranquilizantes afectó el rendimiento académico. Conclusiones: es importante continuar realizando estudios similares, a fin de obtener resultados más precisos y esclarecer la relación entre consumo de drogas y rendimiento académico, por lo que se recomienda incorporar variables relacionadas con las consecuencias del consumo en indicadores como asistencia, entrega de tareas y calificaciones.

Palabras clave: consumo de drogas, Instituciones de Educación Superior, rendimiento escolar. 


\title{
Drugs and Academic Performance among Health Sciences Students at Two Latin American Universities
}

\begin{abstract}
Introduction: Drug use among the young population of developing countries has increased in the last decades; educational and health institutions are concerned about this trend, as it can alter learning and memory, and particularly affects university students. Purpose: To describe and compare the relationship between drug use and academic performance among Health Sciences students at two Latin American universities. Method: Non-probability convenience sampling was used and the Drug Use among University Students Questionnaire (CODEU) was applied online. Data analysis was carried out based on descriptive statistics, and the point biserial correlation coefficient and Kruskal-Wallis non-parametric test were applied, considering the grade average obtained during the last period studied. Results: At the Universidad Veracruzana it was found that the use of alcohol, marijuana, cocaine and inhalable drugs decreases academic performance, while at the Universidad de Boyacá only the use of tranquilizers affected academic performance. Conclusions: It is important to continue conducting similar studies in order to obtain more accurate results and to clarify the relationship between drug use and academic performance. Therefore, it is recommended that variables related to the consequences of drug use be incorporated into indicators such as attendance, homework, and grades.
\end{abstract}

Keywords: drug use, Higher Education Institutions, academic performance.

\section{Drogas e desempenho acadêmico em estudantes da área de saúde de duas universidades latino-americanas}

\section{Resumo}

Introdução: o consumo de drogas na população jovem dos países em desenvolvimento tem aumentado durante as últimas décadas; as instituições de ensino e de saúde estão preocupadas com isso, já que pode alterar a aprendizagem e a memória particularmente dos estudantes universitários. Objetivo: descrever e comparar a relação entre consumo de drogas e desempenho acadêmico em estudantes da área de ciências da saúde de duas universidades latino-americanas. Metodologia: utilizou-se uma amostra não probabilística por conveniência e aplicou-se de forma on-line o questionário Consumo de Drogas em Estudantes Universitários (Codeu). A análise da informação foi realizada com base em estatísticas descritivas e aplicou-se o coeficiente de correlação ponto-bisserial e o teste não paramétrico de Kruskal-Wallis, considerando a média de qualificações obtida durante o último período cursado. Resultados: na Universidad Veracruzana (México), constatou-se que o consumo de álcool, maconha, cocaína e inaláveis diminui o desempenho acadêmico; na Universidad de Boyacá (Colômbia), somente o consumo de tranquilizantes o afetou. Conclusões: é importante continuar realizando estudos semelhantes a fim de obter resultados mais exatos e esclarecer a relação entre consumo de drogas e desempenho acadêmico; portanto, recomenda-se incorporar variáveis relacionadas com as consequências do consumo em indicadores como presença, entrega de tarefas e qualificações.

Palavras-chave: consumo de drogas, Instituições de Ensino Superior, rendimento escolar. 


\section{Introducción}

Diversos reportes internacionales -como el Informe Mundial sobre Drogas 2015 y los estudios realizados por el Proyecto de Apoyo a la Comunidad Andina en el Área de Drogas Sintéticas (Drosican) y el Programa Anti-Drogas Ilícitas (Pradican)-, al igual que diferentes encuestas nacionales -como la Encuesta Nacional de Adicciones en México (2011) y el Estudio Nacional de Consumo de Sustancias Psicoactivas en Colombia 2013 (2014)-, permiten establecer que en los países en vías de desarrollo se registra un incremento en el consumo de drogas y todos coinciden en: i) que este aumento se observa en la población juvenil y en mujeres, y ii) que se registra una disminución en la edad de inicio.

Para efectos de este trabajo, se consideró a la población universitaria de licenciatura y tecnicatura entre 18 y 25 años de edad, por lo que el estudio se realizó en dos Instituciones de Educación Superior (IES) latinoamericanas, una universidad pública de México y una privada de Colombia, en las que se analiza la relación entre consumo de drogas y rendimiento académico.

Los aportes de las neurociencias han mostrado que el cerebro de los adolescentes y jóvenes menores de 25 años no ha alcanzado su plena madurez en el área de la corteza prefrontal, lo que afecta el dominio de ímpetus y la elección de resoluciones, de modo que la presencia de alguna droga, y en particular el alcohol, puede alterar su funcionalidad cuando el consumo se inicia a edades tempranas (Rodríguez et al., 2015). A diferencia del cerebro de los adultos, en el de los adolescentes se producen cambios más profundos que repercuten en la memoria y el aprendizaje, y estos pueden mantenerse por varios días, lo que los hace más duraderos (Instituto Nacional sobre el Abuso de Drogas [NIDA], 2014).

En México hay varias encuestas (Encuesta Nacional de Adicciones 2002, 2008 y 2011, y Encuesta Escolar de Adicciones 2012) que evidencian incrementos importantes en el consumo de drogas en la población juvenil, sobre todo de alcohol y marihuana, en estudiantes de secundaria (ahora comprendido en el básico) del nivel medio superior y superior, al igual que en el caso de las mujeres, cuyo consumo de alcohol y tabaco casi se equipara al de los hombres. A su vez, en Colombia, el Estudio Nacional de Consumo de Sustancias Psicoactivas 2013 muestra un incremento respecto al 2008 en el consumo de alcohol y drogas ilegales en la población de 18 a 24 años.
La mayor parte de los estudios sobre consumo de drogas realizados en población universitaria reporta que las sustancias de mayor consumo son el alcohol, el tabaco y la marihuana -Alcántara et al. (2011), Pradican (2013) y Comisión Interamericana para el Control del Abuso de Drogas-Organización de los Estados Americanos (Cicad-oEA, 2015), entre otros-.

El ingreso a la educación superior se considera una etapa en la que los jóvenes incurren en diferentes conductas de riesgo (Trejo y Murillo, 2012), por ejemplo, el consumo de sustancias psicoactivas. Para entender esta situación, se han construido numerosas explicaciones entre las que destacan: el contacto con entornos permisivos; llegar a la edad legal para el consumo de alcohol y tabaco; la presión del grupo de pares (y en algunos casos, de familiares cercanos); la accesibilidad y disponibilidad de las sustancias legales e ilegales para su consumo; e incluso el cambio del lugar de residencia y el abandono del hogar familiar (derivado de la necesidad de migrar a las ciudades en las que se encuentran las IEs), lo cual en muchas ocasiones supone una disminución de la supervisión parental y el encuentro con otras normas de conducta (Beverido et al., 2016). Así también lo ha señalado el NIDA (2004, p. 9) en Estados Unidos: "Cuando los adultos jóvenes dejan sus hogares para ir a la universidad o para trabajar y se encuentran solos por primera vez, su riesgo para el abuso de drogas y del alcohol es muy alto".

Desde principios de los años ochenta, varias investigaciones -como las realizadas por Brook et al. (1981), Alvira (1986), O'Conell y Patterson (1989) y Chau y Oblitas (2007) - han dado cuenta del consumo de sustancias legales e ilegales en este grupo poblacional. De hecho, algunas reportan que entre el nivel educativo y el consumo hay una asociación positiva (Velásquez y Scoppetta, 1998). Se trata de un asunto preocupante, ya que el consumo en esta etapa se asocia al bajo rendimiento académico, la deserción escolar, la depresión e incluso al suicidio (Kosky, Silburn y Zubrick, 1990).

Al corroborar que se trata de una preocupación mundial, se encontraron trabajos que, sin profundizar mucho en el tema, plantean que el adecuado aprovechamiento académico de los estudiantes es un componente preciso e importante para el desarrollo del individuo en otros campos (Schweinhart et al., 1985; Hernández et al., 2009), y que el consumo de drogas está asociado a un mayor ausentismo, a la deserción y a bajas calificaciones (Kandel, 1985; Elzo et al., 1992, citados en González-Sánchez et al., 1999). 
En investigaciones más recientes, se encontraron aquellas que exploran la asociación entre consumo de drogas y rendimiento académico. En el ámbito internacional, se destaca el trabajo de FontMayolas, Gras y Planes (2006), cuya muestra estuvo conformada por 584 estudiantes de la Universidad de Gerona (España) y reportó asociación del consumo de cannabis con las peores notas de rendimiento académico y con una mayor prevalencia del abandono de los estudios.

Otro estudio realizado en España por GarridoGonzález, Bugarín-González y Machín-Fernández (2016), en el que participaron 185 estudiantes de la Escuela Universitaria de Enfermería de Lugo, reportó la frecuencia y la cantidad del uso de drogas en estos jóvenes y su asociación con: ser hombre, consumir cigarrillos y bebidas alcohólicas, no vivir con la familia, tener bajas calificaciones y beber en la vía pública; sin embargo, no se encontró relación con la práctica deportiva ni con la lectura.

Una investigación realizada por Ortega-Pérez, Costa-Júnior y Pereira (2011), en una muestra no probabilística de 500 estudiantes del Centro de Salud de Bienestar Universitario de la Universidad Nacional de El Salvador, mostró la manera en que los estudiantes consumidores perciben si las drogas afectan su desempeño escolar: 110 de ellos señalaron que no, 51 consideraron muy bajo el efecto, 19 admitieron que sí afecta en gran medida, 10 reconocieron que por usar drogas no habían logrado aprobar algunos cursos y ninguno admitió haber abandonado los estudios a causa de su consumo. Sin embargo, la percepción en cuanto a sus compañeros consumidores se modificó, de tal modo que 123 opinaron que el consumo sí les afecta de manera importante, ya que han reprobado o desertado de la escuela, y solo 61 estudiantes consideraron como escasa o nula la afectación por el uso de drogas.

Años después, Aguiar et al. (2013) publicaron un estudio realizado en una universidad brasileña con 343 estudiantes, en el que reportan que el patrón predominante de consumo de alcohol (44\%) fue binge drinking (bebedor alto, de acuerdo con la Organización Mundial de la Salud [oms], 1994), con el que los estudiantes presentaron un riesgo 9,2 veces más alto de no concluir sus estudios en el tiempo esperado y eventualmente ver afectado su desarrollo académico.

En sentido contrario, el trabajo de Devi et al. (2013) con estudiantes provenientes de Malasia que cursan Medicina en una facultad de India, cuyo objetivo es conocer el consumo de alcohol, sus predictores y el rendimiento académico, mostró que no existe una asociación entre consumo de alcohol con esta última variable $\left(\chi^{2}=1,34 \mathrm{p}=0,72\right)$, ni cambios en las actividades de ocio entre consumidores de alcohol y no consumidores, ya que ambos grupos prefirieron participar en redes sociales, navegar en internet, y practicar deportes y otras actividades extracurriculares. Lo mismo sucede en el trabajo de Ansari, Stock y Mills (2013), realizado en una universidad de Reino Unido con 379 estudiantes, que estableció que la frecuencia de consumo de alcohol no tiene relación significativa con el aprovechamiento académico.

Como resultado de la revisión de antecedentes en los ámbitos nacionales de los dos países, destaca el estudio realizado en México por Anaya-Ocampo et al. (2006) en el estado de Morelos, en el cual se establece que, al margen de otros factores, la persistencia del tabaquismo está asociada a un deficiente desempeño escolar, aunque no existen diferencias entre fumadores y no fumadores en lo que corresponde al coeficiente intelectual.

Otro estudio realizado por Magaña (2007), en el que participaron 291 estudiantes de segundo a octavo semestre de la Facultad de Enfermería de la Universidad Autónoma de Baja California, con el objetivo de determinar la asociación entre consumo de drogas ilícitas y rendimiento académico, utilizó las pruebas estadísticas de tipo ordinal D de Somers y Tau-b de Kendall, que indicaron que no existe asociación entre las variables en relación con el rendimiento académico y el consumo de drogas ilícitas.

También en México, el estudio de Caso y Hernández (2007), en el que participaron 1581 estudiantes de entre 15 y 23 años de edad, mostró que el consumo de sustancias adictivas como tabaco, alcohol y cocaína registró el coeficiente estandarizado más elevado con respecto a las otras variables predictoras del bajo rendimiento académico consideradas en el análisis de regresión.

Así mismo, un trabajo realizado por Beverido et al. (2015), en el que se compara el consumo de drogas y su relación con el rendimiento académico en una universidad de Veracruz con otra del estado de Puebla, encontró que los alumnos que no consumen drogas presentaron mejor aprovechamiento académico.

En Colombia, la revisión permitió identificar un estudio realizado por Vélez y Roa (2005) en la Facultad de Medicina de la Universidad del Rosario en Bogotá, en el que se encontró que haber fumado marihuana fue, junto con otras conductas, un factor de riesgo para el fracaso académico. Sin embargo, el mismo estudio muestra que esta conclusión no aplica 
para todas las drogas, ya que en el caso del alcohol se encontraron 18 estudiantes con sospecha de alcoholismo y seis en riesgo, pero solo cinco de ellos registraron bajo rendimiento académico, por lo que no hubo asociación estadísticamente significativa.

Otro estudio fue el realizado por Velásquez y Mejía (2009) en la Universidad de Antioquia, que exploró esta misma asociación mediante un análisis factorial relacionando el promedio de calificaciones de los estudiantes con los cursos en los que están inscritos, con los cursos en los que se han dado de baja, con los cursos que han acreditado y con su situación en el semestre que están cursando. Los hallazgos refieren que la probabilidad de bajo rendimiento académico se presenta en los dependientes al cannabis y en los que consumen alcohol de manera riesgosa. Otro estudio realizado por Castaño-Pérez y Calderón-Vallejo (2014), en estudiantes de la Universidad Luis Amigó, reportó que los problemas que resultaron estadísticamente significativos entre faltar o retrasarse en llegar a clase y el consumo perjudicial o dependencia de alcohol fue de $37,3 \%$ y $67,4 \%$ respectivamente; y el menor aprovechamiento académico fue de $16,4 \%$ en el caso de abuso y $52,2 \%$ para dependencia.

Recapitulando, hay numerosos trabajos que establecen una relación entre consumo de drogas legales, médicas sin prescripción e ilegales y rendimiento académico en estudiantes universitarios, y hay otros (más escasos) que niegan que se trate de un elemento que deba ser tomado en cuenta, por lo menos para ciertas drogas. Además, resulta interesante observar las diferencias regionales y sociales del comportamiento de este fenómeno, razón por la que este estudio retoma el primer escenario como hipótesis a comprobar.

\section{Método}

Se hicieron los diagnósticos sobre percepción, riesgo y consumo de drogas en dos universidades latinoamericanas - una pública y una privada- utilizando la misma metodología, y se compararon los resultados en el área de Ciencias de la Salud usando un muestreo no probabilístico por conveniencia. Los criterios de comparación fueron los siguientes: a) ambas son IEs; b) en los dos casos solo se consideró a los estudiantes de la misma área académica; c) ambos grupos consideran el mismo rango de edad (entre $18 \mathrm{y}$ 25 años); d) se utilizó la misma metodología; y e) se aplicó el mismo instrumento en línea.

\section{Participantes}

La Universidad Veracruzana es una IEs pública que en 2012 contaba con una población total en el área de Ciencias de la Salud de 12159 estudiantes, distribuidos en cinco sedes; para este estudio solo se consideraron 1332 estudiantes de la región Xalapa, con una edad promedio de 20,7 años ( $D E=2,29$ ). Por su parte, la Universidad de Boyacá es una IEs privada, distribuida en tres sedes que, al momento de la encuesta, contaba con un total de 1477 estudiantes del área de Ciencias de la Salud, de los cuales participaron en este estudio 524, con una edad promedio de 21,2 años $(\mathrm{DE}=3,39)$. En total, se consideraron 1856 estudiantes del área de Ciencias de la Salud de ambas instituciones. De los 1332 estudiantes de la Universidad Veracruzana, hubo 353 hombres $(26,5 \%)$ y $979 \mathrm{mu}-$ jeres $(73,5 \%)$ participantes; mientras que de los 524 estudiantes de la Universidad de Boyacá, hubo 84 hombres $(16,0 \%)$ y 140 mujeres $(84,0 \%)$ participantes

El proyecto, impulsado por la Universidad Veracruzana, fue evaluado y aprobado por el Consejo Técnico del Instituto de Ciencias de la Salud, por los cuerpos académicos que forman parte de la Red Veracruzana de Investigación en Adicciones (Reviva), por la Defensoría de Derechos Universitarios y por las autoridades universitarias. En ambas universidades, se consideraron los principios éticos de beneficencia, no maleficencia, justicia y autonomía, además de la participación anónima de los estudiantes.

\section{Instrumentos}

El instrumento utilizado fue el Cuestionario sobre Consumo de Drogas en Estudiantes Universitarios (CODEu; Beverido et al., 2012), producto de una adaptación autorizada del Cuestionario de Estudiantes, un instrumento diseñado en el Instituto Nacional de Psiquiatría Ramón de la Fuente Muñiz (2009) y aplicado previamente en la Universidad Nacional Autónoma de México.

El CODEu está integrado por 73 ítems subdivididos en cinco secciones que indagan acerca de: a) aspectos sociodemográficos de los participantes; b) consumo de drogas lícitas, médicas sin prescripción e ilícitas; c) factores y percepción de riesgo sobre el consumo; d) discapacidad asociada al consumo de drogas; y e) percepción respecto a la eficacia de las campañas gráficas para la prevención al alcance de la población estudiantil. 
El cuestionario recibió la validez de contenido a través de la revisión de un panel de tres expertos, definido "como una opinión informada de personas con trayectoria en el tema, que son reconocidas por otros como expertos cualificados en este, y que pueden dar información, evidencia, juicios y valoraciones" (Escobar-Pérez y Cuervo-Martínez, 2008, p. 29), y se cumplió con los criterios para seleccionar expertos propuestos por Skjong y Wentworth (2000), a saber: 1) conocimiento $y$ opiniones sustentadas en grados académicos para realizar arbitrajes, 2) reconocimiento de academias prestigiosas, 3 ) deseo y disposición para participar y 4) honradez, equidad y autoconfianza. Así mismo, el instrumento fue piloteado con una muestra de 70 estudiantes, lo que permitió aplicar la prueba de confiabilidad alfa de Cronbach, obteniendo un valor de 0,787 que equivale a aceptable.

Para la aplicación del instrumento en línea, se utilizó la plataforma LimeSurvey en la Universidad Veracruzana y la MonkeySurvey en la Universidad de Boyacá; en ambos casos, se utilizó el paquete IBM SPSs Statistics, versión 15.

\section{Variables}

Las variables empleadas en este trabajo fueron: consumo de alcohol, consumo de drogas ilegales y rendimiento académico. Para la variable de consumo de alcohol, se tomaron en cuenta tres categorías definidas por la oms: i) el Consumo Moderado o de Riesgo Bajo, que se refiere a la ingesta de cantidades que no causan problemas, es decir, no más de cuatro copas por ocasión y no más de dos veces por semana; ii) el Consumo Abusivo o Bebedor Alto, que se define como el consumo que supera el volumen establecido, correspondiente a cinco copas o más con la misma frecuencia utilizada en la categoría anterior; y iii) el Consumo de Riesgo, que es aquel que excede las cinco copas de tres a seis veces por semana o a diario, y se refiere a un patrón de consumo que eleva las probabilidades de adquirir la adicción y de sufrir consecuencias nocivas que afectan los planos físico, mental y social (oms, 1994).

En la variable de consumo de drogas ilegales, se incluyen las cuatro sustancias psicoactivas de mayor consumo en ambas IEs en el último año y en el último mes.

En cuanto a la variable de rendimiento académico, conviene puntualizar que, para efectos de este trabajo, se define como la evaluación del conocimiento adquirido en el ámbito universitario, medido de acuerdo con el promedio de las calificaciones obtenidas en los exámenes. En este trabajo, se usaron dos escalas de calificaciones: la de la Universidad Veracruzana oscila de dos a 10 puntos, mientras que la de la Universidad de Boyacá va de uno a cinco puntos, por lo que las calificaciones reprobatorias son menores que seis y tres, respectivamente.

El rendimiento académico puede ser analizado desde distintas perspectivas, y como puntualizan López et al. (1996, p. 563), constituye "[...] un problema en sí mismo, el cual está influido por una serie de circunstancias psicológicas, sociales y familiares, entre otros". Por eso, resulta de suma importancia obtener evidencia científica sobre el uso de sustancias y su relación con el conocimiento adquirido por los universitarios, lo que permitirá diseñar programas tanto preventivos como de atención.

El análisis inicial de la información se hizo con base en estadísticas descriptivas, con las que se obtuvieron tablas comparativas por IEs para el consumo de drogas. La prueba estadística chi-cuadrado se empleó para determinar diferencias significativas en el consumo de drogas durante el último año y el último mes en ambas instituciones.

Para comprobar la hipótesis, se utilizó la prueba no paramétrica Kruskall-Wallis, con el fin de determinar diferencias significativas en el rendimiento académico por patrón de consumo de alcohol en ambas IEs. Finalmente, para conocer la relación del consumo de alcohol y drogas ilegales con el rendimiento académico, se calculó el coeficiente de correlación biserial puntual.

\section{Resultados}

En las poblaciones estudiadas, el consumo moderado de alcohol alcanzó más del 65\%; sin embargo, el consumo de riesgo se encuentra por arriba en la IEs de Boyacá con respecto a la de Veracruz (16\% y 9,5\%, respectivamente).

En cuanto al consumo de drogas ilegales, las tablas 1 y 2 ilustran el consumo de las drogas más usadas por los estudiantes de las dos universidades en el último año y en el último mes. Esto proporciona información sobre el consumo actual, pero no necesariamente hace referencia a un consumo habitual y menos aún a adicción, puesto que este indicador también puede incluir a consumidores experimentales. 
Tabla 1

Consumo de drogas ilegales en el último año

\begin{tabular}{lccc}
\hline \multirow{2}{*}{ Tipo de droga } & \multicolumn{2}{c}{ IEs } & \multirow{2}{*}{ p-value } \\
\cline { 2 - 3 } & Veracruz & Boyacá & \\
\hline Tranquilizantes & 1,7 & 3,1 & 0,419 \\
Marihuana $^{*}$ & 8,3 & 1,9 & 0,001 \\
Cocaína & 1,1 & 0,2 & 0,534 \\
Inhalables & 0,2 & - & - \\
\hline
\end{tabular}

Nota. ${ }^{*}$ Diferencias significativas entre ambas instituciones. Chi-cuadrado, $p<0,05$. codeu Veracruz-Boyacá 2012

El consumo de marihuana en el último año presentó diferencias significativas en ambas instituciones (tabla 1): en la universidad colombiana fue de $1,9 \%$ y en la IEs de Veracruz fue de $8,3 \%$. El consumo registrado en la universidad mexicana supera notablemente el registrado en la Encuesta Nacional de Adicciones - ENA 2011 (1,9\%) en población entre 18 y 34 años. Por el contrario, los resultados en la Universidad de Boyacá fueron menores a los encontrados en el proyecto Pradican (2013), en el que la población universitaria mostró un consumo de $15,2 \%$.

En relación con el indicador de consumo de drogas ilegales durante el último mes (tabla 2), el estudio mostró que la droga de mayor consumo nuevamente fue la marihuana con $3,9 \%$ en la Universidad Veracruzana, mientras que en la Universidad de Boyacá la droga de mayor consumo fueron los tranquilizantes con $1,9 \%$. Una vez más, se encontró una diferencia estadísticamente significativa entre ambas instituciones para el caso de la marihuana.

Tabla 2

Consumo de drogas en el último mes

\begin{tabular}{lccc}
\hline \multirow{2}{*}{ Tipo de droga } & \multicolumn{2}{c}{ IES } & p-value \\
\cline { 2 - 3 } & Veracruz & Boyacá & \\
\hline Tranquilizantes & 0,9 & 1,9 & 0,432 \\
Marihuana* $_{\text {Cocaína }}^{3,9}$ & 0,8 & 0,024 \\
Inhalables & 0,4 & -- & -- \\
\hline
\end{tabular}

Nota. ${ }^{*}$ Diferencias significativas entre ambas instituciones. Chi-cuadrado, $p<0,05$. codeu Veracruz-Boyacá 2012

La tabla 3 muestra que el consumo abusivo y de riesgo en ambas IEs no afectó el promedio de calificaciones, lo que resultó diferente a lo esperado. La prueba estadística no paramétrica Kruskall-Wallis confirma que no existen diferencias significativas entre el rendimiento académico y el patrón de consumo de alcohol en los estudiantes en la IEs de Veracruz $(\mathrm{H}=4,712$, $\mathrm{p}<0,095)$ y tampoco en el caso de la IEs de Boyacá $(\mathrm{H}=0,281, \mathrm{p}=0,869)$.

Tabla 3

Patrón de consumo de alcohol y promedio de calificaciones

\begin{tabular}{lcc}
\hline \multirow{2}{*}{ Consumo de alcohol } & \multicolumn{2}{c}{ IEs } \\
\cline { 2 - 3 } & Veracruz & Boyacá \\
\hline & Promedio de calificaciones \\
\hline Consumo de riesgo & 8,4 & 3,73 \\
Consumo abusivo & 8,4 & 3,75 \\
Consumo moderado & 8,6 & 3,72 \\
\hline
\end{tabular}

Nota. CODEU Veracruz-Universidad de Boyacá 2012

Para determinar la asociación entre rendimiento académico y consumo de drogas en el último año, se utilizó el coeficiente de correlación biserial puntual (tabla 4), el cual se emplea cuando las variables han sido medidas de diferente manera, es decir, una como variable dicotómica (sí/no consume) y la otra en escala de intervalos (promedio de calificaciones).

Tabla 4

Relación entre consumo de drogas y rendimiento escolar de acuerdo con el coeficiente de correlación biserial puntual

\begin{tabular}{lcccc}
\hline \multirow{2}{*}{ Tipo de droga } & \multicolumn{4}{c}{ IEs } \\
\cline { 2 - 5 } & Veracruz & $\mathrm{p}$-value & Boyacá & $\mathrm{p}$-value \\
\hline Alcohol & $-4,161$ & 0,097 & 0,042 & 0,338 \\
Tranquilizantes & 0,012 & 0,574 & $-0,039$ & 0,381 \\
Marihuana & $-0,021$ & 0,375 & 0,021 & 0,591 \\
Cocaína & $-0,047$ & 0,094 & 0,053 & 0,171 \\
Inhalables & $-0,032$ & 0,205 & -- & -- \\
\hline
\end{tabular}

El nivel de significancia considerado fue $<0,05$. CODEU Veracruz-Universidad de Boyacá 2012

Si bien el índice de correlación biserial puntual fue bajo y los resultados no expresan significancia estadística, se puede observar que hay menor rendimiento en los estudiantes de Veracruz que consumieron sustancias psicoactivas, excepto con los tranquilizantes que muestran un valor positivo. En contraste, en el caso de la IEs de Boyacá, el rendimiento académico se vio afectado únicamente por el consumo de tranquilizantes. 


\section{Discusión}

Como se mencionó antes, existen muchos trabajos que relacionan el consumo de drogas y el rendimiento académico. Sin embargo, los resultados no son concluyentes, ya que unos muestran relación y otros señalan que el consumo no influye en el rendimiento; además, es importante examinar las diferencias entre poblaciones, tiempo y espacio en el comportamiento de este fenómeno. A pesar de que este trabajo estableció la hipótesis que relaciona el consumo y el rendimiento académico, los resultados obtenidos no permiten demostrarlo.

En consideración a las razones anteriores, a continuación se exponen las diferencias y las semejanzas entre los hallazgos de este trabajo y las investigaciones revisadas. En lo que corresponde al análisis del consumo durante el último año y el último mes, de lo que parte el análisis de este trabajo, la comparación de los diagnósticos realizados en la Universidad Veracruzana (México) y en la Universidad de Boyacá (Colombia) con otros trabajos muestra semejanzas con aquellos realizados por Alcántara et al. (2011), Pradican (2013) y Cicad-oea (2015), los cuales señalan que las drogas más consumidas en el ámbito universitario son el alcohol, el tabaco y la marihuana.

Los resultados sobre la relación consumo de drogas-rendimiento académico muestran que en el caso del alcohol no hay diferencias significativas entre consumo moderado, abusivo y de riesgo y el promedio de calificaciones en ambas IEs. Este resultado coincide con lo registrado en otros estudios, como el de Vélez y Roa (2005) en la Universidad del Rosario (Colombia), el de Devi et al. (2013) en India con estudiantes provenientes de Malasia y el de Ansari et al. (2013) en una universidad de Reino Unido, en los cuales no se encontró una asociación entre consumo de alcohol y rendimiento académico.

Por el contrario, este trabajo presenta diferencias con estudios como el de Hernández et al. (2009) con estudiantes españoles, el de Velásquez y Mejía (2009) en Colombia y el de Aguiar et al. (2013) con estudiantes brasileños, los cuales reportaron que consumir bebidas alcohólicas y otras sustancias psicoactivas sí repercute en el rendimiento académico. El trabajo de Velásquez y Mejía precisa que el consumo de riesgo de alcohol genera mayor probabilidad de presentar bajo rendimiento académico, y el de Aguiar et al. establece que existe un riesgo de no concluir los estudios en el tiempo esperado que es 9,2 veces más alto en los estudiantes que registran un patrón de consumo explosivo de alcohol o binge drinking.

Con respecto al consumo de drogas ilegales y su relación con el rendimiento académico, en el trabajo de Garrido-González et al. (2016) sí encontraron relación. Sin embargo, conviene precisar que en el caso de la IEs mexicana las drogas ilegales que mostraron una tendencia a afectar de manera negativa el rendimiento académico fueron la marihuana, la cocaína y los inhalables, mientras que en la IEs colombiana únicamente se observó este comportamiento en el uso de tranquilizantes consumidos sin prescripción médica.

En el caso específico de la marihuana, los trabajos de Velázquez y Mejía (2009) y de Font-Mayolas et al. (2006) coinciden con los hallazgos en la Universidad Veracruzana, así como en el caso de la cocaína, cuya relación también fue encontrada en el trabajo de Caso y Hernández (2007); en el consumo de ambas drogas se detectó una relación con el bajo rendimiento académico. Por el contrario, el trabajo de Magaña (2007) no encontró asociación entre ambas variables.

Los resultados de esta investigación no son generalizables para otras poblaciones estudiantiles, debido a que no se abordó una muestra representativa de estudiantes de ambas instituciones participantes. Otra limitación consiste en que no se consideraron factores como el consumo diferenciado por sexo y otros elementos que forman parte del estudio del rendimiento académico, tales como el pénsum de cada institución, la asistencia, la entrega de tareas y el tiempo para la conclusión de los estudios, aspectos que podrían ser abordados en investigaciones posteriores.

\section{Conclusiones}

Se cumplió con el objetivo establecido de estudiar el consumo de drogas y su asociación con el rendimiento académico en las dos universidades que participaron en la investigación. El trabajo comparativo aporta al conocimiento de este fenómeno en ámbitos donde no había sido estudiado, es decir, en la Universidad Veracruzana y en la Universidad de Boyacá.

Ante este y otros riesgos implicados en el consumo de drogas, adquiere relevancia la formación integral de los estudiantes que promueva el desarrollo de habilidades protectoras del consumo de drogas y el establecimiento de relaciones interpersonales sanas.

Las acciones preventivas en el ámbito de la educación superior deberán incluir información sobre el riesgo que representa el consumo de alcohol y otras 
drogas en el adecuado desarrollo académico, y promover la construcción dentro de las universidades de espacios enriquecidos para el desarrollo de actividades deportivas y culturales que contribuyan a un estilo de vida saludable.

\section{Referencias}

Aguiar, A. S., Fausto, M. A., Da Silva-Fonseca, V. A., Haddad, M. y Quintaes K. D. (2013). Pattern of alcoholic beverage consumption and academic performance among college students. Revista de Psiquiatría Clínica, 40(2), 65-70. doi: 10.1590/S0101-60832013000200003

Alcántara, A., Cuétara, C., Pérez, J. y Pulido M. A. (2011). Consumo de drogas y alcohol en universidades privadas de dos ciudades mexicanas. Psicología y Salud México, 21(1), 39-46.

Alvira, F. R. (1986). Cambios en el consumo de bebidas alcohólicas en España. Revista Española de Investigaciones Sociológicas, 34(86), 111-130.

Anaya-Ocampo, R., Arillo-Santillán, E., Sánchez-Zamorano, L. M. y Lazcano-Ponce, E. (2006). Bajo desempeño escolar relacionado con la persistencia del tabaquismo en una cohorte de estudiantes en México. Salud Pública de México, 48(supl1), S17-S29. doi: 10.1590/S003636342006000700004

Ansari, W. E., Stock, C. y Mills, C. (2013). Is alcohol consumption associated with poor academic achievement in university students? International Journal of Preventive Medicine, 4(10), 1175-1188.

Beverido, P., Salas, B., De San Jorge, X., Pavón, P., Blázquez, M. S., Gogeascoechea, M. C. y Cruz, A. (2012). Cuestionario sobre Consumo de Drogas en Estudiantes Universitarios (CODEU). Xalapa: Universidad Veracruzana.

Beverido, P., Salas, B., De San Jorge, X. y Cruz, A. (2015). Bajo rendimiento académico: ¿resultados de un consumo precoz? Revista de Investigación y Desarrollo, 1(1), 56-66.

Beverido, P., Salas, B., De San Jorge, X. y Avendaño, Y. (2016). La migración interna en estudiantes universitarios. ¿Un riesgo para el consumo de drogas? Ciencia UAT, 10(2), 23-32.

Brophy, J. y Good, T. (1984). Teacher behavior and student achievement. Michigan: Institute for Research for Teaching. Recuperado de http://files.eric.ed.gov/fulltext/ ED251422.pdf

Brook, M. L., Walfish, S., Stenmark, D. E. y Canger, J. M. (1981). Personality variables in alcohol abuse in college students. Journal of Drug Education, 11(2), 185-189. doi: 10.2190/9QKG-P0C8-P1C9-WVMB
Caso, J. y Hernández, L. (2007). Variables que inciden en el rendimiento académico de adolescentes mexicanos. Revista Latinoamericana de Psicología, 29(3), 487-501.

Castaño-Pérez, G. A. y Calderón-Vallejo, G. A. (2014). Problemas asociados al consumo de alcohol en estudiantes universitarios. Revista Latino-Americana de Enfermagem, 22(5), 739-746. doi: 10.1590/01041169.3579.2475

Chau, C. y Oblitas H. (2007). Características del consumo de bebidas alcohólicas en estudiantes universitarios. Revista Peruana de Drogodependencias, 5(1), 43-61.

Comisión Interamericana para el Control del Abuso de Drogas-Organización de los Estados Americanos (Cicad-oEA). (2015). Informe sobre uso de drogas en las Américas. Washington: Observatorio Interamericano de Drogas. Recuperado de http://www.cicad.oas.org/ apps/Document.aspx? $\mathrm{Id}=3209$

Devi, V., Wei Ping, A. T., Tuan Ying, T., Subhash, S. y Mahmood Hafez, S. (2013). Alcohol use, its predictors and academic performance among Malaysian students of a medical college in India. Education in Medicine Journal, 5(4), 72-76. doi: 10.5959/eimj.v5i4.155

Escobar-Pérez, J. y Cuervo-Martínez, A. (2008). Validez de Contenido y Juicio de Expertos: una aproximación a su utilización. Avances en Medición, 6, 27-36.

Font-Mayolas, S., Gras, M. E. y Planes, M. (2006). Análisis del patrón de consumo de cannabis en estudiantes universitarios. Adicciones, 18(4), 337-344.

Garrido-González, I., Bugarín-González, R., Machín-Fernández, A. (2016). Consumo de drogas en estudiantes de enfermería. Enfermería Clínica, 26(3), 174-180. doi: 10.1016/j.enfcli.2015.11.006

Gobierno Nacional de la República de Colombia. (2014). Estudio Nacional de Consumo de Sustancias Psicoactivas en Colombia 2013. Informe Final. Bogotá: ALvI Impresores. Recuperado de https://www.unodc.org/ documents/colombia/2014/Julio/Estudio_de_Consumo_UNODC.pdf

González-Sánchez, J. D., Guerra-Lobera, L. I., Díaz-Negrete D. B. y Arellanez-Hernández, J. (1999). Adherencia escolar y consumo de drogas. México D. F.: Centros de Integración Juvenil, Subdirección de Investigación. Recuperado de http://www.cij.gob.mx/Programas/ Investigacion/pdf/ps3-99-07.pdf

Hernández, T., Roldán, J., Jiménez, A., Mora, C., Escarpa, D. y Pérez, M. T. (2009). La edad de inicio en el consumo de drogas, un indicador de consumo problemático. Psychosocial Intervention, 18(3), 199-212. 
Instituto Nacional de Psiquiatría Ramón de la Fuente Muñiz. (2009). Cuestionario de Estudiantes 2009. Recuperado de https://goo.gl/MvMLBR

Instituto Nacional sobre el Abuso de Drogas. (NIDA). (2004). Cómo prevenir el uso de drogas en los niños y los adolescentes. Bethseda: Institutos Nacionales de la Salud.

Instituto Nacional sobre el Abuso de Drogas. (NIDA). (2014). Las drogas, el cerebro y el comportamiento: la ciencia de la adicción. Recuperado el 11 de septiembre de 2017, de https:// www.drugabuse.gov/es/publicaciones/las-drogas-el-cerebro-y-el-comportamiento-la-ciencia-de-la-adiccion

Kosky, R., Silburn, S. y Zubrick, L. (1990). Are children in adolescence who have suicidal thoughts different from those who attempt suicide? Journal of Nervous Mental Disorders, 178, 38-43 doi. 10.1111/j.1469-7610.1997.tb01611.x

López, E. K., Medina-Mora, M. E., Villatoro, J., Juárez, F. y Berenzon, S. (1996). Factores relacionados al consumo de drogas y al rendimiento académico en adolescentes. La Psicología Social en México, 6, 561-568.

Magaña, A. (2007). Relación del consumo de drogas ilícitas con el rendimiento académico (tesis de maestría, Universidad Autónoma de Querétaro, México).

Medina-Mora, M. E., Cravioto, P., Villatoro-Velázquez, J. A., Galván, F., Fleiz-Bautista, C., Rojas E., et al. (2003). Encuesta Nacional de Adicciones 2002. México D. F.: Consejo Nacional contra las Adicciones e Instituto Nacional de Psiquiatría Ramón de la Fuente Muñiz.

Medina-Mora, M. E., Villatoro-Velázquez, J. A., RodríguezAjenjo, C., Gutiérrez, M. L., Moreno, M. I., Fleiz-Bautista, C., et al. (2009). Encuesta Nacional de Adicciones 2008. México D. F.: Instituto Nacional de Salud Pública.

Medina-Mora, M. E., Villatoro-Velázquez, J. A., Fleiz-Bautista, C., Téllez-Rojo, M. M., Mendoza-Alvarado. L. R., Romero-Martínez, M., et al. (2012). Encuesta Nacional de Adicciones 2011: Reporte de Drogas. México D. F.: Instituto Nacional de Psiquiatría Ramón de la Fuente Muñiz-Instituto Nacional de Salud Pública y Secretaría de Salud. Disponible en: http://www.conadic.salud. gob.mx/pdfs/ENA_2011_DROGAS_ILICITAS_.pdf

O’Conell, D. y Patterson, H. (1989). A survey of current college alcohol abuse: Programs, attitudes and training needs. Journal of Alcohol and Drug Education, 43(2), 61-69.

Oficina de las Naciones Unidas contra la Droga y el Delito. (2016). Informe Mundial sobre Drogas 2015. Resumen Ejecutivo. Recuperado de https://goo.gl/cDZ5PD
Organización Mundial de la Salud (oms). (1994). Glosario de Términos de Alcohol y Drogas. Madrid: Ministerio de Sanidad y Consumo de España. Recuperado de https://goo.gl/p5YsX4

Ortega-Pérez, C. A., Costa-Júnior, M. L. y Pereira, G. (2011). Perfil epidemiológico de la drogadicción en estudiantes universitarios. Revista Latino-Americana de Enfermagem, 19, 665-672.

Programa Anti-Drogas Ilícitas en la Comunidad Andina (Pradican) (2013). Proyecto Pradican. II Estudio Epidemiológico Andino sobre Consumo de Drogas en Población Universitaria. Informe Regional 2012. Secretaría General de la Comunidad Andina. Lima: Cicad-oeA.

Rodríguez, S., Corral, M., Doallo, S. y Cadaveira, F. (2015). Funcionamiento neurocognitivo. En M. T. Cortés (coord.), Consumo intensivo de alcohol en jóvenes. Guía Clínica (pp. 73-94). Barcelona: Sociodrogoalcohol.

Schweinhart, L. J., Berrueta-Clement, J. R., Barnett, W. S., Epstein, A. E. y Weikart, D. P. (1985). Effects of the Perry Preschool Program on youths through age 19. Topics in Early Childhood Special Education, 5(2), 26-35. doi: 10.1177/027112148500500204

Skjong, R. y Wentworth, B. H. (2000). Expert judgement and risk perception. Det Norske Veritas. Recuperado de https://goo.gl/cqohLy

Trejo Cuevas, E. y Murillo López, S. (2012). Consulta Juvenil sobre Adicciones en el Distrito Federal. Resumen Ejecutivo. México D. F.: Instituto para la Prevención y Atención de las Adicciones en la Ciudad de México, Universidad Nacional Autónoma de México.

Velásquez, C. y Mejía, J. (2009). Rendimiento académico y abuso-dependencia de sustancias psicoactivas en los estudiantes de la Universidad de Antioquia. Memorias IV CLABEs: Cuarta Conferencia Latinoamericana sobre el Abandono en la Educación Superior. Recuperado de https://goo.gl/cBvgou

Velásquez, J. y Scoppetta, O. (1998). Consumo de sustancias psicoactivas en estudiantes de carreras técnicas y tecnológicas de Santa Fe de Bogotá, 1997. Bogotá: Secretaría Distrital de Salud. Recuperado de https://goo.gl/ BwbeuT

Vélez, A. y Roa, C. (2005). Factors associated with academic performance in medical students. Educación Médica, 8(2), 24-32. 